\title{
The MarR Repressor of the Multiple Antibiotic Resistance (mar) Operon in Escherichia coli: Prototypic Member of a Family of Bacterial Regulatory Proteins Involved in Sensing Phenolic Compounds
}

\author{
Mark C. Sulavik, Laura F. Gambino, and Paul F. Miller \\ Infectious Diseases Section, Therapeutics Department, Parke-Davis \\ Pharmaceutical Research Division, Warner-Lambert Company, \\ Ann Arbor, Michigan, U.S.A.
}

\begin{abstract}
Background: The marR gene of Escherichia coli encodes a repressor of the marRAB operon, a regulatory locus controlling multiple antibiotic resistance in this organism. Inactivation of $m a r R$ results in increased expression of $\operatorname{mar} A$, which acts at several target genes in the cell leading to reduced antibiotic accumulation. Exposure of $E$. coli to sodium salicylate (SAL) induces marRAB operon transcription and antibiotic resistance. The mechanism by which SAL antagonizes MarR repressor activity is unclear.

Materials and Methods: Recombinant plasmid libraries were introduced into a reporter strain designed to identify cloned genes encoding MarR repressor activity. Computer analysis of sequence databases was also used to search for proteins related to MarR.

Results: A second E. coli gene, MprA, that exhibits MarR
\end{abstract}

repressor activity was identified. Subsequent database searching revealed a family of 10 proteins from a variety of bacteria that share significant amino acid sequence similarity to MarR and MprA. At least four of these proteins are transcriptional repressors whose activity is antagonized by SAL or by phenolic agents structurally related to SAL.

Conclusions: The MarR family is identified as a group of regulatory factors whose activity is modulated in response to environmental signals in the form of phenolic compounds. Many of these agents are plant derived. Some of the MarR homologs appear more likely to control systems expressed in animal hosts, suggesting that phenolic sensing by bacteria is important in a variety of environments and in the regulation of numerous processes.

\section{INTRODUCTION}

In Escherichia coli, a simultaneous increase in resistance to a number of structurally unrelated antibiotics is associated with mutations at the multiple antibiotic resistance (mar) locus, located at $34^{\prime}$ on the E. coli chromosome (1). Susceptibility to a number of antibiotic classes is affected by this system, including tetracycline, chloram-

Address correspondence and reprint requests to: Paul F. Miller, Infectious Diseases Department, Parke-Davis Pharmaceutical Research, 2800 Plymouth Road, Ann Arbor, MI 48105 , U.S.A. phenicol, $\beta$-lactams, and fluoroquinolones $(2,3)$. Reduced drug accumulation, resulting from activation of efflux systems along with down-regulation of outer membrane porins, appears to be the primary resistance mechanism (2-5). A Mar phenotype has also been observed in a variety of gram-negative, quinolone resistant clinical isolates, suggesting that nonspecific multidrug resistance systems may play a role in the outcome of antibiotic therapy regimens with this class of antibiotics (6).

Recent efforts from several labs have helped clarify the identity and function of genes en- 


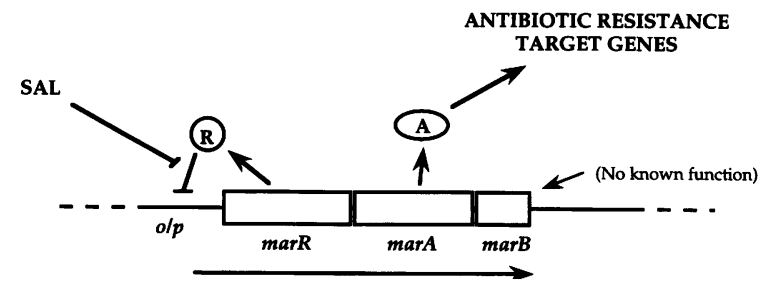

FIG. 1. Genetic organization of the marRAB operon

MarR is an autorepressor that normally maintains mar operon expression at a low level. In the presence of inducers such as salicylate (SAL), MarR repressor activity is antagonized, resulting in increased levels of MarA and, consequently, increased antibiotic resistance.

coded at the mar locus that play a role in the resistance scheme. These studies have drawn attention to a small operon encoding the marRAB genes as the critical element in this system (Fig. 1). This locus appears to be regulatory in nature. marA encodes a positive regulator of antibiotic resistance whose level of expression seems to be the critical determinant of antibiotic resistance. A Tn 5 insertion in $\operatorname{mar} A$ reverses the resistant phenotype of mar mutants (1), and overexpression of this gene confers resistance even in a strain containing a large deletion of the mar region of the chromosome (7). MarA shares significant amino acid sequence similarity to members of the AraC family of transcriptional activators $(7,8)$, most notably SoxS, a positive regulator of the superoxide stress response whose induction also leads to a Mar phenotype $(9,10)$.

Control of marRAB operon transcription, and thus of marA expression, is the role of the marR gene product. Mutations that result in increased antibiotic resistance are frequently located in $\operatorname{marR}(8,11)$, and $\operatorname{marR}$ is necessary to maintain marRAB operon expression at a low level $(11,12)$. These findings indicate that MarR is a negative regulator of the marRAB operon, and imply that marRAB operon expression is inducible.

Support for this notion has recently emerged. A conditional antibiotic resistant phenotype similar to that associated with mar mutants can be elicited by culturing $E$. coli in the presence of the aromatic weak acid sodium salicylate (SAL) (13). Moreover, exposure to SAL results in the rapid induction of marRAB operon transcription and of expression of a mar-lacZ fu- sion, providing a partial explanation for the effects of this agent (14). Strains deleted for the mar locus are defective in SAL-inducible antibiotic resistance (14), and the only genes encoded at the mar locus that are required for the salicylate effect are marR and marA (12). A recent report indicated that a hybrid protein consisting of a fusion between maltose binding protein and MarR can bind the marRAB promoter region in vitro, and that this binding is antagonized by a variety of chemical agents including SAL (15). These observations suggest a simple model in which MarR directly represses marRAB operon transcription, and SAL antagonizes MarR repressor activity, presumably by dissociating MarR from the operator region (Fig. 1). Since exposure to acetylsalicylic acid (aspirin) can induce the same antibiotic resistance phenotype (13), an awareness of the importance of the mar locus during infection and antibiotic therapy seems warranted.

Thus, an understanding of the physiological signals governing MarR repressor activity would be very informative with respect to the control of intrinsic antibiotic resistance in enteric organisms. We report here the finding that MarR is related, both in primary amino acid sequence and in function, to a family of bacterial regulatory proteins that are involved in specific responses to environmental phenolic compounds. This discovery significantly expands the context in which the mar system should be considered and suggests that the marRAB operon is part of a larger stimulon that may be important in virulence and resistance processes in bacteria.

\section{MATERIALS AND METHODS}

\section{Bacterial Strains and Plasmids}

All strains described in this study are E. coli $\mathrm{K} 12$ derivatives of MC4100 $\left(\mathrm{F}^{-}\right.$araD139 $\Delta($ lac $) U 169$ rpsL relA thi) (16). Strain B318, used extensively herein, contains the $\Delta 1738$ deletion (17) which removes $\sim 39 \mathrm{kbp}$ of DNA from the mar region of the chromosome including the marRAB operon. It is also lysogenic for a recombinant lambda phage carrying a marR-lacZ protein fusion, in which lac $Z$ is fused in frame after the 75th codon of $m a r R$, and expression of the fusion is under the control of the marRAB operon promoter (12). Expression of this fusion is constitutive in strain B318 due to the absence of a source of MarR repressor. 
E. coli and Salmonella typhimurium plasmid libraries were constructed from size-selected fragments of Sau3A partial digests of chromosomal DNA from either strain W3110 (E. coli) or SR-11 (S. typhimurium [18]), cloned into the BamHI site of pBR322 (for E. coli [19]) or pBluescript $\mathrm{SK}^{-}$(for $S$. typhimurium; Stratagene). pmarR (12) consists of a $570 \mathrm{bp}$ PCR fragment containing the marRAB operon operator/promoter region and all of marR, cloned into pBR322. The pS plasmids shown in Fig. 2 were constructed by subcloning the indicated restriction fragments of pmarD (see text) into pBluescript $\mathrm{SK}^{-}$(Stratagene). pmprA was constructed by PCR synthesis of the mprA gene using primers based on the published sequence (20). The vector was pBluescript $\mathrm{SK}^{-}$. The mprA::cat allele was constructed by first isolating the cat gene from $\lambda 1324$ (21) by digestion of the phage DNA with BamHI, converting the BamHI sites into SphI sites using linkers, and cloning the fragment into the SphI site in mprA in plasmid pS6 (Fig. 2). This results in disruption of $m p r A$ after the 34th codon. The mprA::cat plasmid was linearized by digestion with PstI and used to replace the normal mprA gene by linear transformation of a recD::Tn 10 strain (22). Subsequent strains containing the mprA::cat allele were constructed by P1 transduction. pEcl7kd was constructed by PCR synthesis of a DNA fragment corresponding to the coding region of the $17 \mathrm{kd}$ gene from the prs locus (23) using chromosomal DNA from strain ECOR 55 (24) as a template. This fragment was then cloned into the IPTG-inducible expression plasmid pTrc99A (Pharmacia, Piscataway, $\mathrm{NJ})$.

\section{Reagents and Growth Conditions}

Bacteria were propagated in LB media (25), which, when needed, was solidified with $1.5 \%$ Bacto-Agar (DIFCO, Detroit, MI). All antibiotics were from Sigma Chemical Co. (St. Louis, MO, U.S.A.) other reagents were from commercial sources and were of the highest purity available.

\section{Other Genetic and Biochemical Methods}

DNA hybridizations were performed following standard protocols (26). Probes were labeled with digoxigenin using the Genius kit (Boehringer-Mannheim, Indianapolis, IN) following the manufacturers instructions. For Southern hybridizations, DNA samples were resolved on agarose gels and transferred to Nytran membranes
(Schleicher and Schuell, Keene, NH) by capillary transfer following protocols from the membrane supplier. The miniset blot of the ordered recombinant phage collection of Kohara (27) was from TaKaRa. $\beta$-Galactosidase assays were performed as described by Miller (25) using the chloroformSDS method of cell permeabilization. Briefly, cultures were grown in L broth to an $A_{600}=0.3$, at which time inducers were added to $2.5 \mathrm{mM}$. Incubation was continued for $1 \mathrm{hr}$, and $\beta$-galactosidase activity, expressed as Miller Units, was determined.

\section{Computer Methods}

Alignments of promoter regions shown in Fig. 3 were performed using MacVector version 4.5 software for the Macintosh (Eastman Kodak Co., Rochester, NY, U.S.A.). The computer searches that generated the sequences shown in Fig. 4 were performed using TFASTA (28) in the GCG package of sequence analysis programs (29). Initial searches used MarR or MprA as the query sequence. New matches found in these searches were then used as queries in additional searches. This iterative process was repeated until no new members were found. Alignment of the sequences was performed using the PILEUP program (29).

\section{RESULTS \\ Identification of a Functional Homolog of marR}

In the course of genetic studies on the regulation of mar operon transcription, we constructed an E. coli strain, B318, which proved useful for cloning mar homologs from related enteric organisms. B318 contains a 39-kbp deletion of the mar region of the chromosome including the $\operatorname{mar} R A B$ operon, and also carries a $\lambda$ phage containing a marR-lacZ translational fusion under the control of the mar operator/promoter region (12). Expression of this fusion is constitutively derepressed $\left(\mathrm{Lac}^{+}\right)$in this strain due to the absence of MarR repressor. Introduction of a plasmid containing marR restores both repression of fusion expression and inducibility by SAL (12). The presence of marA on the same plasmid restores normal levels of SAL-inducible antibiotic resistance (14).

Our initial objective was to use B318 to clone mar sequences from virulent enteric organisms, 


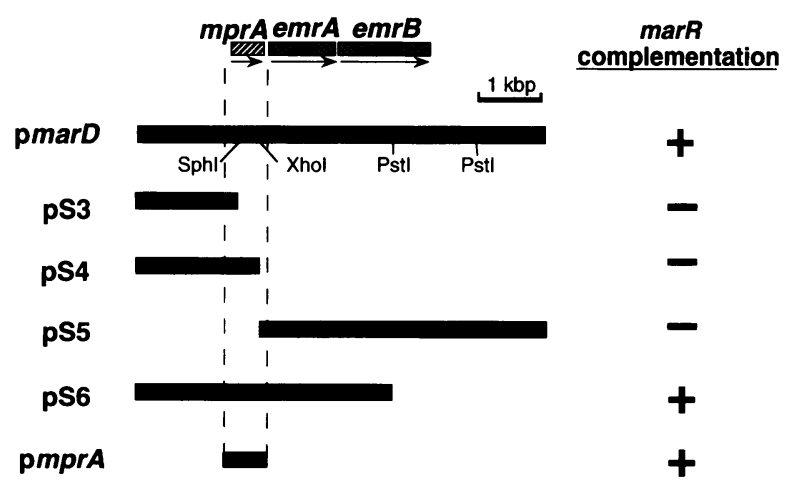

FIG. 2. Restriction map and complementation data for marD plasmids

The restriction map for $\mathrm{pmar} D$ and the positions of the $m p r A$, emrA, and $e m r B$ genes are shown at the top. pmarD is pBluescript $\mathrm{SK}^{-}$(Stratagene) derivative containing a $6.0-\mathrm{kb}$ fragment from a random SauIIIA digest of chromosomal DNA of $E$. coli strain W3110. Specific subclones were generated as described in Materials and Methods. marR complementation indicates the ability of the specified plasmid to restore a $\mathrm{Lac}^{-}$phenotype on MacConkey-lactose media to strain B318.

and then construct deletion strains for the purpose of testing the importance of the mar system in various pathogenic models. When B 318 was transformed with a plasmid library containing random S. typhimurium chromosomal DNA, nine white $\left(\mathrm{Lac}^{-}\right)$transformants were obtained on MacConkey-lactose indicator plates. These were further tested for the presence of marRAB operon DNA by Southern blotting. Two classes of clone were identified. The first class (five transformants) contained sequences that hybridized with an E. coli mar probe, and included the $S$. typhimurium marR gene as judged by DNA sequence analysis. The remaining four transformants contained cloned DNA sequences that were not detected by the E. coli mar probe, but cross-hybridized with each other, indicating that they were derived from the same locus and possibly encoded a second mar repressor. This new marRlike activity was designated marD.

To determine if $E$. coli also had a marD gene the cloning strategy was repeated, this time using an E. coli plasmid library. White transformants were again identified and, as in the Salmonella experiment, the population was mixed. Fourteen of fifteen clones were shown by hybridization to carry mar sequences. The remaining isolate contained a cloned insert that cross-hybridized with the $S$. typhimurium marD fragment, indicating that this locus was also present in E. coli. The
TABLE 1. Regulation of marR-lacZ by MarR and MprA

\begin{tabular}{lccc}
\hline & & \multicolumn{2}{c}{$\begin{array}{c}\boldsymbol{\beta} \text {-Galactosidase } \\
\text { Activity } \\
\text { Following } \\
\text { Induction with: }\end{array}$} \\
\cline { 3 - 4 } Plasmid & Background $^{a}$ & $\begin{array}{c}\text { No } \\
\text { Addition }\end{array}$ & SAL $^{b}$ \\
\hline None & $W T$ & 15 & 300 \\
None & $\Delta m a r$ & 420 & 730 \\
pmarR & $\Delta m a r$ & 5 & 150 \\
pmprA & $\Delta m a r$ & 10 & 250 \\
& & & \\
\hline
\end{tabular}

${ }^{a}$ All experiments were performed in strain B318 except for the first entry, which was in an isogenic strain containing a wild-type mar locus.

${ }^{b} \mathrm{SAL}$, sodium salicylate.

corresponding plasmid was designated pmarD (Fig. 2).

To find the location of marD on the E. coli chromosome, $\mathrm{pmarD}$ was used as a hybridization probe against the Kohara miniset blot (27). Positive hybridization signals localized the cloned sequence to the $52^{\prime}$ region of the chromosome. Comparison of the restriction map for this region of the chromosome with that of $\mathrm{p} m a r D$ indicated that three known genes, $m p r A$, emrA, and emrB, were present within the $6 \mathrm{kbp}$ cloned segment (Fig. 2). To localize marD repressor activity within this interval, deletion and subcloning experiments were performed. As is shown in Figure 2, this activity was associated with the mprA gene, previously identified as a repressor of genes involved in the biosynthesis of the peptide antibiotic microcin B17 $(20,30)$.

One feature of MarR repressor activity is its antagonism by SAL (14). To determine if MprAmediated repression of marR-lacZ was similarly affected, B318 derivatives carrying either $m p r A$ or marR plasmids were treated with SAL and assayed for $\beta$-galactosidase activity. As is shown in Table 1, SAL relieved repression mediated by either marR or mprA. These results indicate that $m p r A$, when present on a multicopy plasmid, can functionally replace mar $R$ and restore regulation to a marR-lacZ fusion.

It seemed unlikely that $m p r A$ played a significant role in regulating mar gene expression in wild type cells, since a deletion of $\operatorname{mar} R$ alone 
results in high level derepression of a marR-lacZ fusion. To determine if $m p r A$ played a minor role in regulating mar gene expression, a chloramphenicol resistance determinant was inserted after the 34th codon of the cloned mprA gene, and the disrupted allele was recombined onto the chromosome in place of the normal gene (see Materials and Methods). In an otherwise wildtype strain (i.e., $\operatorname{mar}^{+}$), the $m p r A:$ :cat allele had no affect on marR-lacZ fusion expression. In addition, expression of this fusion in a strain containing a marR::Tn 10 allele was also unaffected by the introduction of mprA::cat (data not shown). Thus, marR and $m p r A$ are not redundant regulators in the $E$. coli strains used here. The complementation of a marR deficiency by $m p r A$ requires that the latter be present in multicopy.

In wild-type cells, MprA is known to repress expression from two specific promoters: that of the microcin $\mathrm{B} 17$ biosynthetic gene cluster $\left(\mathrm{P}_{m c b A}\right)(30)$ and its own promoter $\left(\mathrm{P}_{m p r A}\right)(20)$. A recent report indicated that $m p r A$ expression is significantly induced following salicylate treatment (31). To confirm that this is the case in the strain background used here, we measured expression of a mprA-lacZ transcriptional fusion present in single copy on a $\lambda$ prophage under conditions identical to those used to follow marRlacZ fusion expression; namely, 1-hr exposure to $2.5 \mathrm{mM}$ SAL using cells grown to mid-log prior to SAL addition. A 5-fold induction of fusion enzyme expression was consistently observed under these conditions (data not shown). Thus, SAL stimulates the expression of a normal cellular target of MprA.

MarR and MprA may thus act as sequencespecific repressors. As such, promoters controlled by these regulators may contain conserved sequences at appropriate positions with respect to known or suspected recognition sites for RNA polymerase (32). A comparison of the promoter regions for mprA (20) and $m c b A$ (33) revealed such a sequence that was identical at 19 of 24 positions (Fig. 3A). In $\mathrm{P}_{m p r A}$, this element is located $5 \mathrm{bp}$ upstream from the predicted -35 sequence. In contrast, this same sequence is found $77 \mathrm{bp}$ upstream from the -35 element of a promoter known to direct the post-exponential expression of $m c b A$ (33). It seemed possible that the increase in $m c b A$ expression observed in an $m p r A$ mutant during exponential growth results from activation of a different promoter element than that used for postexponential expression. Consistent with this notion, good -35 and -10 elements could be found in the vicinity of the
A.

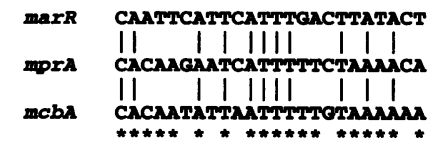

B

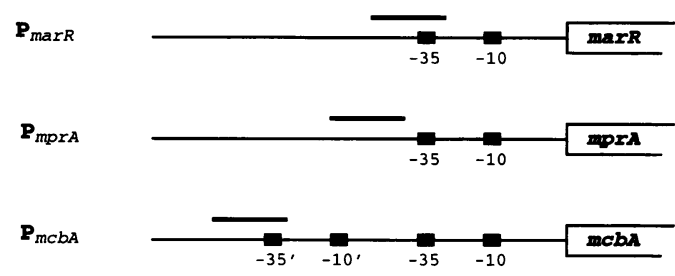

FIG. 3. Identification of a conserved sequence element among the promoters for marR, mprA, and $\boldsymbol{m c b A}$

(A) Alignment of conserved sequences. Vertical lines indicate nucleotide positions that are identical among all three sequences. Asterisks beneath the $m c b A$ sequence show residues that are identical in $m p r A$ and $m c b A$. (B) Positions of conserved elements in the promoter regions of $m a r R, m p r A$, and $m c b A$. Conserved sequences aligned in Panel $\mathrm{A}$ are indicated by solid bars.

putative MprA recognition sequence $\left(-35^{\prime}\right.$ and $-10^{\prime}$ in Fig. 3B). The $-35^{\prime}$ was buried within the putative MprA recognition sequence.

Comparison of the promoter regions of marR (8) and $m p r A$ indicated that the region of greatest similarity corresponded to the conserved sequence found in the $\mathrm{P}_{m p r A} / \mathrm{P}_{m c b A}$ alignment. Figure $3 \mathrm{~A}$ shows that the $\mathrm{P}_{\text {marR }}$ element is identical at 11 of 24 positions with the other two sequences. Moreover, the putative -35 sequence of $\mathrm{P}_{\text {marR }}$ is nested within this conserved element (Fig. 3B).

\section{Identification of Other Bacterial Homologs of MarR}

Previous computer-assisted searches had failed to identify any significant similarities between MarR and translated sequences in the available databases (8). However, an alignment of MarR and MprA showed that the two proteins share $28 \%$ identity at the amino acid sequence level. Inclusion of conservative amino acid replacements increased the similarity to $47 \%$. Since the complementation results (Table 1 ) indicated that this level of similarity is significant and related to function, we reexamined the databases for evidence of other homologs, using the program 


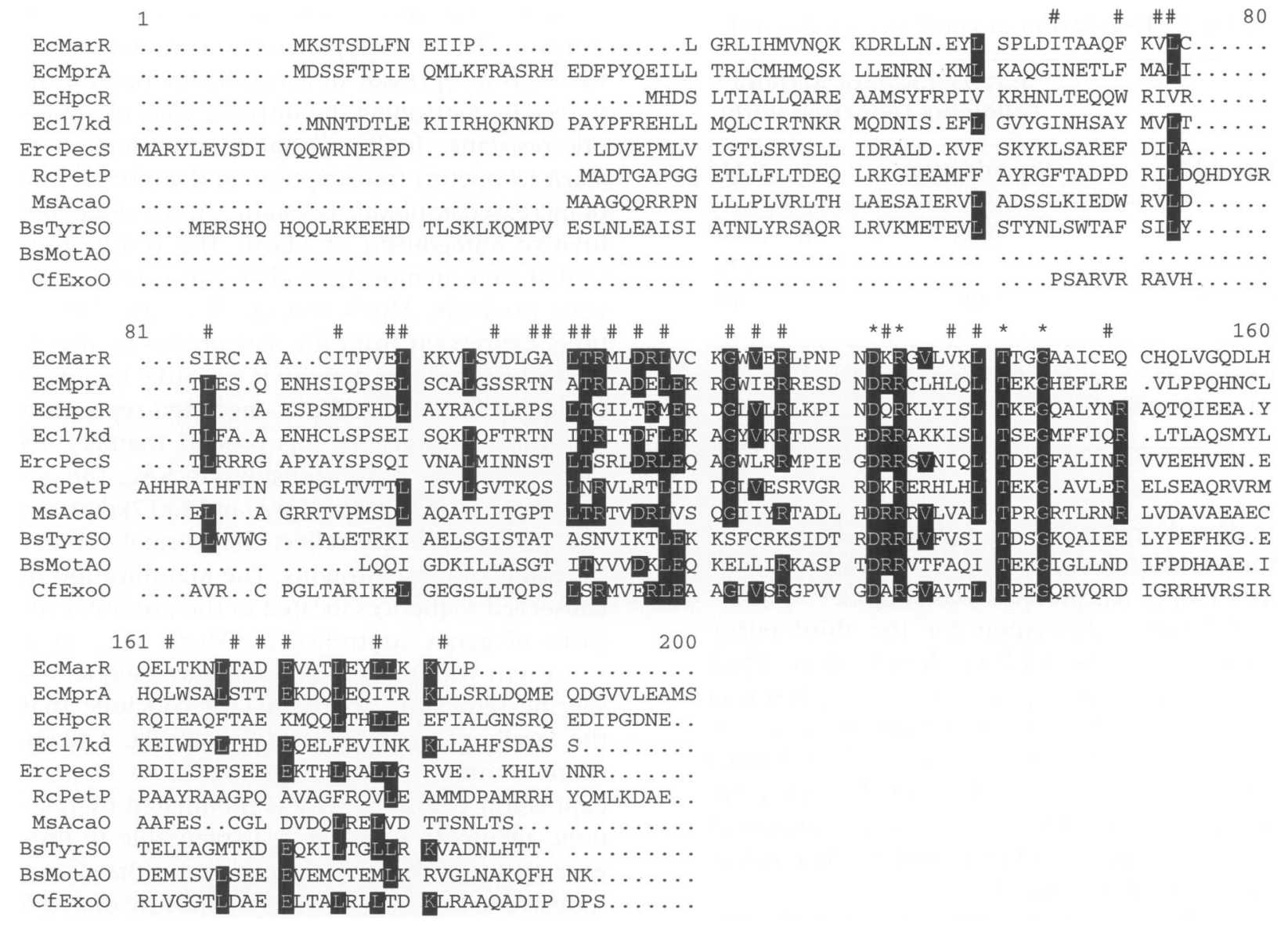

FIG. 4. Alignment of MarR and related proteins

Protein sequences shown were identified using TFASTA to search currently available GenEMBL and SwissProt databases. The alignment was generated using the PILEUP program in the GCG package (29). Entries are not listed in order of similarity, but rather reflect their bacterial origin as described in the text. Amino acids highlighted in black boxes are residues identical at that position for at least five of the proteins. *, the four residues that are identical in all ten sequences; \#, a position in which a chemically conserved amino acid is present in at least 8 of the 10 entries, based on the following groupings of conserved amino acids: YWF, QNED, MILV, PAGST, HKR. Relevant information, GenEMBL accession numbers, and references, respectively, are as follows: EcMarR: MarR from $E$. coli, accession number M96235 (8); EcMprA: MprA from E. coli, X54141 (20); EcHpcR: HpcR from E. coli C strain, S56952 (34); Ecl7kd: 17 kd ORF from prs gene cluster in pyelonephritic E. coli ECOR 55 strain, X62158 (23); ErcPecS: PecS from Erwinia chrysanthemi, X74409 (35); RcPetP: PetP from Rhodobacter capsulatus, Z12113 (36); MsAcaORF: ORF P1 in the entry for Mycobacterium smegmatis acetamidase gene, X57175 (37); BsTyrSORF: ORF3 in the tyrS sequence entry from Bacillus subtilis, X52480 (38); BsMotABORF: 3' end of an ORF downstream from and convergently transcribed towards the motAB genes of $B$. subtilis, M77238 (39); CfExoORF: An ORF upstream of and divergently transcribed from the Cellulomonas fimi exogluconase gene, L1 $1080(40)$. This last open reading frame does not contain a normal initiation codon and may be derived from incorrect sequence information. Additional information on some of the entries is described in the text.

TFASTA (28). This analysis revealed eight additional proteins that were similarly related to MarR (Fig. 4). All of the sequences identified are of bacterial origin, with four entries from $E$. coli strains and the remainder from diverse species including gram-positive, gram-negative and mycobacteria. Except for the extreme amino terminal regions, conserved residues are found distrib- uted throughout the entire length of the alignment. In addition, there is a cluster of highly conserved amino acids extending from residues 111-144 of the alignment that includes four invariant positions. A preliminary consensus sequence of DXRXXXXX(L/I)TXXG (residues 132144) can be compiled that appears to be a signature for this family of proteins. Given the 
TABLE 2. Regulation of marR-lacZ by Ecl7kd

\begin{tabular}{lcc}
\hline & \multicolumn{2}{c}{$\begin{array}{c}\boldsymbol{\beta} \text {-Galactosidase Activity } \\
\text { Following Induction with: }\end{array}$} \\
\cline { 2 - 3 } Plasmid & No Addition & SAL $^{a}$ \\
\hline None & 530 & 960 \\
pmarR & 5 & 170 \\
pEcl7kd & 66 & 310 \\
\hline
\end{tabular}

All experiments were performed in strain B318.

${ }^{a} \mathrm{SAL}$, sodium salicylate.

functional relationship between MarR and MprA, evidence for analogous properties of the other two $E$. coli proteins was sought.

Relevant information for the third entry, EcHpcR, was obtained from the literature. HpcR is found in $E$. coli $\mathrm{C}$ strains and is a repressor of adjacent genes involved in the catabolism of homoprotocatechuate (HPC) (34), a plant-derived phenolic that is structurally related to SAL. Importantly, $\mathrm{HpcR}$ repressor activity is antagonized by HPC (34) in a manner similar to the effect of SAL on MarR and MprA.

Ecl7kd, the fourth entry shown, is the putative product of an open reading frame linked to the pap/prs cluster of genes associated with pilus formation in certain pyelonephritic $E$. coli strains. Although no function has been associated with this ORF as yet, its similarity to MprA has been noted previously (23). In order to determine if Ecl7kd could restore MarR repressor activity to strain B318, the coding region for Ecl7kd was synthesized by PCR and cloned into the IPTGinducible expression plasmid pTrc $99 A$. However, we found that even in the absence of induction with IPTG, the Ecl7kd construct repressed marRlacZ expression in strain B318 (Table 2), suggesting that leaky expression from the trc promoter produced sufficient $17 \mathrm{kd}$ protein to complement the marR deficiency in this strain. Furthermore, this repressor activity was antagonized by SAL. Thus, Ecl7kd is also functionally related to MarR.

\section{DISCUSSION}

The findings presented in this study identify a new family of bacterial regulatory proteins related not only by primary amino acid sequence similarity but also, in the cases tested here, by function. The prototypic member of this family, MarR, is a repressor of a regulatory operon involved in controlling the intrinsic level of antibiotic resistance in $E$. coli. Salicylate induction of marR $A B$ operon transcription, and consequently of increased antibiotic resistance, must somehow involve antagonism of MarR. The results presented here demonstrate that two other $E$. coli gene products, MprA and Ecl7kd, can also repress expression from the mar promoter. Moreover, this repressor activity is sensitive to antagonism by SAL. Since MprA-mediated repression of a normal target for this protein (namely, its own promoter) is also sensitive to SAL, the activities associated with MprA and Ecl7kd at the mar promoter likely reflect the normal cellular functions of these proteins. The identification of conserved sequences located in the promoter regions of genes controlled by MprA (Fig. 3) is consistent with this protein acting as a sequencespecific repressor. Therefore, we conclude that the family of proteins identified in Fig. 4 likely share common functions as regulators of gene expression whose activity is modulated by phenolic agents. In addition, it is plausable to propose that a stimulon exists in $E$. coli that is responsive to SAL (Fig. 5). Thus, exposure of $E$. coli strains to this agent would derepress the expression of genes controlled by MarR and its related repressors, and thus affect the activity of several independently regulated genetic pathways. Such a treatment would result in an increase in antibiotic resistance (MarR controlled) and microcin B17 antibiotic production (MprA controlled), along with the target genes controlled by Ecl7kd, should the particular strain contain the pap/prs cluster. The proximity of $\operatorname{mpr} A$ to the downstream emrA and $e m r B$ genes, which encode an efflux pump for xenobiotic agents (42), is similar to the physical arrangement of marR and $\operatorname{mar} A$, and raises the possibility that expression of the EmrAB pump is also controlled by MprA. The cellular target(s) for Ecl7kd regulation is not known; however, its association with a virulence locus suggests that it may participate in a pathogenic function. Since the mar locus is known to be present in a large number of enteric species (43) and the experiments reported here indicate that $m p r A$ is also found in $S$. typhimurium, it seems reasonable to propose that the SAL stimulon is conserved among the enterics. SAL has also been reported to induce increased antibiotic resistance in Pseudomonas cepacia, sug- 


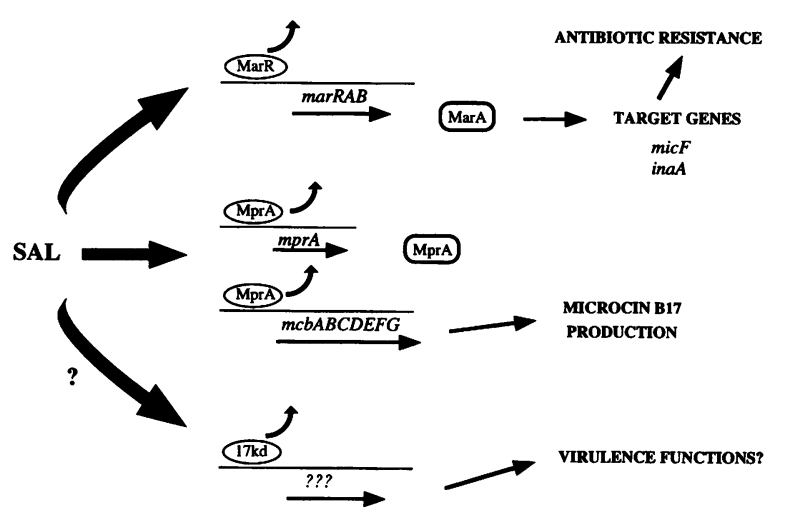

FIG. 5. The SAL stimulon

MarR and its relatives MprA and Ecl7kd are transcriptional repressors acting at specific promoters. In the presence of SAL, the repressing activity of these proteins is antagonized, allowing transcription of the regulated operons. For the MarR and MprA pathways, the consequences of derepression are shown at the right. micF encodes an antisense mRNA that acts to repress production of the major outer membrane porin OmpF, and is induced in mar mutants (3). inaA is a weak acid-inducible gene whose expression is stimulated by MarA (4l). For Ecl 7kd, the target(s) of repression have not been identified; the question mark next to the arrow leading from SAL to $17 \mathrm{kd}$ indicates that antagonism of $17 \mathrm{kd}$-mediated repression of a normal target promoter has not been demonstrated. Other MarR homologs are predicted to carry out similar activities in controlling the expression of specific target genes.

gesting that drug resistance associated with sensing of this phenolic is widespread (44).

Although not specifically related to pathogenesis, the identification of $\mathrm{HpcR}$ as a member of this family may help clarify other issues regarding the mechanism of repressor antagonism. $\mathrm{HpcR}$ is known to regulate expression of a catabolic pathway that allows soil-dwelling $E$. coli C strains to metabolize homoprotocatechuate (HPC) $(34,45)$. As HPC is thought to be the authentic inducer for the $h p c$ system, the possibility that SAL is structurally related to the natural inducer of the mar system is raised. The observation that the mar and $h p c$ systems have nothing in common other than their respective repressors and inducers is consistent with the concept of direct recognition of the inducer by the repressor, as has been suggested for MarR (15).

The remaining proteins listed in Fig. 4 are from bacteria other than $E$. coli. These include gram-positive and gram-negative species as well as mycobacteria, indicating that MarR family members are not restricted to enterics. The PecS protein from Erwinnia chrysanthemi (ErcPecS) is associated with the transcriptional control of pectate lyase and cellulase production, which are involved in invasion of plant tissues (35). petP from Rhodobacter capsulatus is part of a two-gene operon with a second putative transcriptional regulator petR, a genetic organization similar to that of marR and marA (36). The remaining proteins are inferred from open reading frames present in database entries, but for which no genetic information exists. However, since the non- $E$. coli members of this family exhibit similar levels of sequence conservation and include the same highly conserved residues as the $E$. coli proteins, a reasonable expectation is that they are also regulatory proteins and that their activity is affected by a phenolic compound. The target genes for this latter group remain to be identified, but the finding that they can be associated by sequence similarity with the MarR family should provide a means for their detection. It is worth noting that other as yet unidentified MarR family members may exist in $E$. coli and could have escaped detection in the cloning experiments described here, since the screen we employed demanded that the cloned gene product recognize and repress expression from the marRAB promoter. MarR homologs capable of binding SAL but that have different promoter specificities would not have been identified.

The organisms in which the gene products listed in Fig. 4 are found are largely free-living bacteria or plant pathogens. In addition, SAL is an important signaling molecule in plants (46). It thus seems likely that phenolic sensing is an important component of environmental surveillance by free-living bacteria, particularly when considering the variety of noxious aromatic agents produced by plant species (47). However, the association of Ecl7kd with the MarR family suggests that phenolic sensing may play a role in animal infections as well, since the presence of the pap cluster with which the gene encoding Ecl $7 \mathrm{kd}$ is associated correlates strongly with the pyelonephritic capability of E. coli (23). Thus, MarR family members may also carry out important roles in phenolic sensing in animal hosts, and so might monitor either plant-derived compounds ingested by the host or host-synthesized phenolic metabolites. Such inducers might themselves be toxic compounds against which these organisms need to protect themselves; alternatively, they might serve as cues that the bacterium is in the intestinal environment, and could signal 
the induction of protective (MarR, MprA) and colonization (Ecl7kd) factors. Experiments directed towards determining the importance of specific MarR family members in these processes should be extremely informative in this regard.

Preliminary experiments indicate that there is some specificity with respect to the inducing agent. Syringaldehyde, a substituted phenolic that has been identified as an inducer of virulence gene expression in Agrobacterium tumefaciens (48), is also a potent inducer of the mar system (PFM, unpublished observations). Dinitrophenol has also been shown to efficiently stimulate marRAB operon transcription (14). In contrast, HPC is not an effective inducer of mar expression (PFM and MCS, unpublished observations). Moreover, while SAL is a good inducer of mar expression, salicyl alcohol is not (14). In this light, it is interesting to speculate on the role of the conserved amino acids shared by MarR family members (Fig. 4). These residues could be involved in phenolic binding, operator/ promoter recognition, or protein-protein interactions (e.g. dimerization of repressor molecules, interaction with an accessory factor, etc). Since the MarR homologs are likely to exhibit different specificities for phenolics and promoters, conserved amino acids should be involved in interactions that are carried out in very similar ways among these repressors.

The identity of the natural inducers for most of the proteins in the MarR family remains to be determined. However, the finding that the repressor activities of MarR, MprA, and Ecl7kd can be antagonized by SAL, a plant-derived phenolic, raises the possibility that plant products are the natural ligands for many, if not all, of these systems. For HpcR this is clearly the case. Moreover, syringaldehyde and related compounds were first identified as stimulants of virulence gene expression in Agrobacterium tumefaciens (48); similar signals may be recognized by MarR homologs from other plant pathogens and symbionts (e.g., ErcPecS, RcPetP, CfExoO) and may play a role in regulating virulence gene expression in these organisms. MarR and its relatives found in enteric bacteria such as E. coli (EcMprA, Ecl7kd) might modulate gene expression in response to similar phenolics encountered in the gut of mammalian hosts as products of plant diets. In any case, the results reported here suggest that environmental surveillance of phenolic compounds is highly conserved in bacteria and may be important in a variety of signal transduction pathways.

\section{ACKNOWLEDGMENTS}

The authors would like to thank Leonard Post, Steve Gracheck, and Eric Olson for critically reading the manuscript, two anonymous reviewers for their insightful comments, and to our colleagues in the Infectious Diseases Section and the Olson lab for their support throughout.

\section{REFERENCES}

1. George AM, Levy SB. (1983) Gene in the major cotransduction gap of the Escherichia coli K-12 linkage map required for the expression of chromosomal resistance to tetracycline and other antibiotics. J. Bacteriol. 155: 541-548.

2. George AM, Levy SB. (1983) Amplifiable resistance to tetracycline, chloramphenicol, and other antibiotics in Escherichia coli: Involvement of a non-plasmid-determined efflux of tetracycline. J. Bacteriol. 155: 531540.

3. Cohen SP, McMurry LM, Hooper DC, Wolfson JS, Levy SB. (1989) Cross-resistance to fluoroquinolones in multiple-antibiotic-resistant (Mar) Escherichia coli selected by tetracycline or chloramphenicol: Decreased drug accumulation associated with membrane changes in addition to OmpF reduction. Antimicrob. Agents and Chemother. 33: 1318-1325.

4. Cohen SP, McMurry LM, Levy SB. (1988) marA locus causes decreased expression of OmpF porin in multiple-antibiotic-resistant (Mar) mutants of Escherichia coli. J. Bacteriol. 170: 5416-5422.

5. McMurry LM, George AM, Levy SB. (1994) Active efflux of chloramphenicol in susceptible Escherichia coli strains and in multipleantibiotic-resistant (Mar) mutants. Antimicrob. Agents Chemother. 38: 542-546.

6. Hooper DC, Wolfson JS. (1989) Bacterial resistance to the quinolone antimicrobial agents. Am. J. Med. 87: 17S-23S.

7. Gambino L, Gracheck SJ, Miller PF. (1993) Overexpression of the MarA positive regulator is sufficient to confer multiple antibiotic resistance in Escherichia coli. J. Bacteriol. 175: 2888-2894.

8. Cohen SP, Hachler H, Levy SB. (1993) Genetic and functional analysis of the multiple antibiotic resistance (mar) locus in Escherichia coli. J. Bacteriol. 175: 1484-1492. 
9. Greenberg JT, Monach P, Chou JH, Josephy PD, Demple B. (1990) Positive control of a global antioxidant defense regulon activated by superoxide-generating agents in Escherichia coli. Proc. Natl. Acad. Sci. U.S.A. 87: 6181-6185.

10. Miller PF, Gambino LF, Sulavik MC, Gracheck SJ. (1994) Genetic relationship between soxRS and mar loci in promoting multiple antibiotic resistance in Escherichia coli. Antimicrob. Agents Chemother. 38: 1773-1779.

11. Ariza RA, Cohen SP, Bachhawat N, Levy SB, Demple B. (1994) Repressor mutations in the marRAB operon that activate oxidative stress genes and multiple antibiotic resistance in Escherichia coli. J. Bacteriol. 176: 143148.

12. Sulavik MC, Gambino LF, Miller PF. (1994) Analysis of the genetic requirements for inducible multiple-antibiotic resistance associated with the mar locus in Escherichia coli. J. Bacteriol. 176: 7754-7756.

13. Rosner JL. (1985) Nonheritable resistance to chloramphenicol and other antibiotics induced by salicylates and other chemotactic repellants in Escherichia coli K-12. Proc. Natl. Acad. Sci. U.S.A. 82: 8771-8774.

14. Cohen SP, Levy SB, Foulds J, Rosner JL. (1993) Salicylate induction of antibiotic resistance in Escherichia coli-Activation of the mar operon and a mar-independent pathway. J. Bacteriol. 175: 7856-7862.

15. Seoane AS, Levy SB. (1994) Reversal of MarR binding to the regulatory region of the marRAB operon by structurally unrelated inducers. In: Abstracts of the 94th Annual Meeting of the American Society for Microbiology. American Society for Microbiology, Washington, DC, Abstr. H-26, p. 204.

16. Casadaban MJ. (1976) Transposition and fusion of lac genes to selected promoters in Escherichia coli using bacteriophage lambda and Mu. J. Mol. Biol. 104: 541-555.

17. Hill TM, Henson JM, Kuempel PL. (1987) The terminus region of the Escherichia coli chromosome contains separate loci that exhibit polar inhibition of replication. Proc. Natl. Acad. Sci. U.S.A. 84: 1754-1758.

18. Schneider HA, Zinder ND. (1956) Nutrition of the host and natural resistance to infection. V. An improved assay employing genetic markers in the double strain inoculation test. J. Exp. Med. 103: 207-223.

19. Bolivar F, Rodriguez RL, Greene PJ, et al. (1977) Construction and characterization of new cloning vehicles. II. A multipurpose cloning system. Gene 2: 95-113.

20. del Castillo I, Gonzalez-Pastor JE, San Millan JL, Moreno F. (1991) Nucleotide sequence of the Escherichia coli regulatory gene mprA and construction and characterization of mprA-deficient mutants. J. Bacteriol. 173: 3924-3929.

21. Kleckner N, Bender J, Gottesman S. (1991) Uses of transposons with emphasis on $\operatorname{Tn} 10$. In: Miller JH (ed). Bacterial Genetic Systems. Vol. 204, Abelson JN, Simon MI (eds). Methods in Enzymology. Academic Press, San Diego, pp. 139-180.

22. Russell CB, Thaler DS, Dahlquist FW. (1989) Chromosomal transformation of Escherichia coli $\mathrm{rec} D$ strains with linearized plasmids. $J$. Bacteriol. 171: 2609-2613.

23. Marklund B-I, Tennent JM, Garcia E, et al. (1992) Horizontal gene transfer of the Escherichia coli pap and prs pili operons as a mechanism for the development of tissue-specific adhesive properties. Mol. Microbiol. 6: 22252242.

24. Ochman H, Selander RK. (1984) Standard reference strains of Escherichia coli from natural populations. J. Bacteriol. 157: 690-693.

25. Miller JH. (1972) Experiments in Molecular Genetics. Cold Spring Harbor Laboratory, New York.

26. Ausubel FM, Brent R, Kingston RE (eds). (1989) Current Protocols in Molecular Biology. John Wiley and Sons, New York.

27. Kohara Y, Akiyama K, Isono K. (1987) The physical map of the whole E. coli chromosome: Application of a new strategy for rapid analysis and sorting of a large genomic library. Cell 50: 495-508.

28. Pearson WR, Lipman DJ. (1988) Improved tools for biological sequence comparison. Proc. Natl. Acad. Sci. U.S.A. 85: 2444-2448.

29. Devereux J, Haeberli P, Smithies O. (1984) A comprehensive set of sequence analysis programs for the VAX. Nucleic Acids Res. 12: 387395.

30. del Castillo I, Gomez JM, Moreno F. (1990) $m p r A$, an Escherichia coli gene that reduces growth-phase-dependent synthesis of microcins $\mathrm{B} 17$ and $\mathrm{C} 7$ and blocks osmoinduction of proU when cloned on a high-copynumber plasmid. J. Bacteriol. 172: 347-445.

31. Lomovskaya O, Lewis K, Matin A. (1994) Regulation of the emrAB operon in Escherichia coli. (Poster 184). Molecular Genetics of Bacteria and Bacteriophage. Madison, WI. 
32. Collado-Vides J, Magasanik B, Gralla JD. (1991) Control site location and transcriptional regulation in Escherichia coli. Microbiol. Rev. 55: 371-394.

33. Connell N, Han Z, Moreno F, Kolter R. (1987) An E. coli promoter induced by the cessation of growth. Mol. Microbiol. 1: 195201.

34. Roper DI, Fawcett T, Cooper RA. (1993) The Escherichia coli $\mathrm{C}$ homoprotocatechuate degradative operon: $h p c$ gene order, direction of transcription and control of expression. Mol. Gen. Genet. 237: 241-250.

35. Reverchon S, Nasser W, Robert-Badouy J. (1994) pecS: A locus controlling pectinase, cellulase and blue pigment production in $E r$ winnia chrysanthemi. Mol. Microbiol. 11: 11271139.

36. Tokito MK, Daldal F. (1992) petR, located upstream of the $f b c F B C$ operon encoding the cytochrome $b c_{1}$ complex, is homologous to bacterial response regulators and necessary for photosynthetic and respiratory growth of Rhodobacter capsulatus. Mol. Microbiol. 6: 1645-1654.

37. Mahenthiralingam E, Draper $\mathrm{P}$, Davis EO, Colston MJ. (1993) Cloning and sequencing of the gene which encodes the highly inducible acetamidase of Mycobacterium smegmatis. J. Gen. Microbiol. 139: 575-583.

38. Glaser P, Kunst F, Debarbouille M, Vertes A, Danchin A, Dedonder R. (1991) A gene encoding a tyrosine tRNA synthetase is located near sacS in Bacillus subtilis. DNA Sequence 1: 251-261.

39. Mirel DB, Lustre VM, Chamberlin MJ. (1992) An operon of Bacillus subtilis motility genes transcribed by the $\sigma^{\mathrm{D}}$ form of RNA polymerase. J. Bacteriol. 174: 4197-4204.

Contributed by P. Cuatrecasas on January 20, 1995.
40. O'Neill G, Goh SH, Warren RAJ, Kilburn DG, Miller Jr RC. (1986) Structure of the gene encoding the exoglucanase of Cellulomonas fimi. Gene 44: 325-330.

41. Rosner JL, Slonczewski JL. (1994) Dual regulation of inaA by the multiple antibiotic resistance (Mar) and superoxide (SoxRS) stress response systems of Escherichia coli. $J$. Bacteriol. 176: 6262-6269.

42. Lomovskaya O, Lewis K. (1992) emr, an Escherichia coli locus for multidrug resistance. Proc. Natl. Acad. Sci. U.S.A. 89: 8938-8942.

43. Cohen SP, Yan W, Levy SB. (1993) A multidrug resistance regulatory locus is widespread among enteric bacteria. J. Infect. Dis. 168: 484-488.

44. Burns JL, Clark DK. (1992) Salicylate-induced antibiotic resistance in Pseudomonas cepacia associated with absence of a pore-forming outer membrane protein. Antimicrob. Agents Chemother. 36: 2280-2285.

45. Jenkins JR, Cooper RA. (1988) Molecular cloning, expression, and analysis of the genes of the homoprotocatechuate catabolic pathway of Escherichia coli. J. Bacteriol. 170: 5317-5324.

46. Yalpani N, Raskin I. (1993) Salicylic acid: A systemic signal in induced plant disease resistance. Trends Microbiol. 1: 88-92.

47. Wink M. (1988) Plant breeding: Importance of plant secondary metabolites for protection against pathogens and herbivores. Theoret. Appl. Genet. 75: 225-233.

48. Duban ME, Lee K, Lynn DG. (1993) Strategies in pathogenesis: mechanistic specificity in the detection of generic signals. Mol. Microbiol. 7: 637-645. 